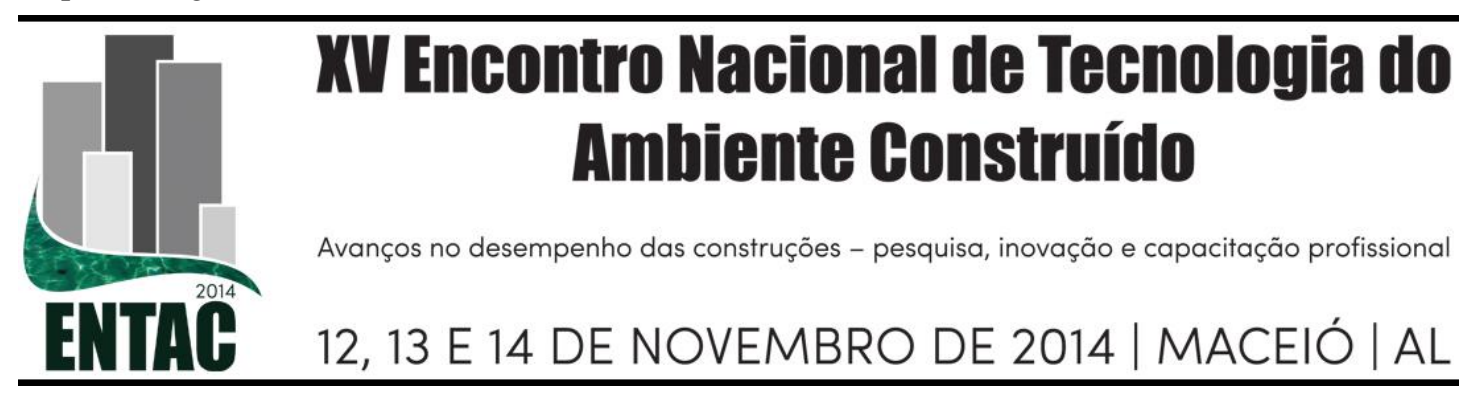

\title{
O USO DE DISPOSITIVOS MÓVEIS PARA FINS DE ACESSIBILIDADE EM AMBIENTE DE ENSINO UNIVERSITÁRIO ENTAC 2014 - MACEIÓ - AL
}

\author{
DIAS, Miriam Carla do Nascimento (1); CASTAÑON, José Alberto Barroso (2) \\ (1) UFJF, (32) 8847-8011, e-mail: arquitetamiriamdias@gmail.com (2) UFJF, \\ e-mail: castanon@terra.com.br
}

\begin{abstract}
RESUMO
Há tempos, o desenvolvimento tecnológico vem influenciando as formas de se ver, pensar e vivenciar a arquitetura. Cada inovação, independente de sua área técnica, traz conectada a si uma reestruturação das técnicas construtivas e consequentemente da paisagem. Todo aprimoramento tecnológico possui a finalidade de melhorar a condição da vida humana, intervindo diretamente na eficácia e na eficiência da execução de suas atividades cotidianas. A humanidade tem presenciado o surgimento das novas técnicas de representação gráfica em ambiente virtual e o desenvolvimento de softwares cada vez mais potentes e dinâmicos. Os hardwares tiveram que acompanhar todo esse desenvolvimento e o surgimento dos modelos sem fio permitiu que o escritório pudesse estar em qualquer lugar, e parte destes hardwares foi transformado em dispositivos móveis como celulares e tablets, já considerados como importantes instrumentos de trabalho na construção civil. Diante deste contexto, esta pesquisa, objetiva efetivar o uso de dispositivos móveis para facilitar a acessibilidade dentro dos padrões de acesso universal, tendo como ferramenta a criação de um protótipo de aplicativo mobile utilizando tecnologia de geolocalização, baseado em estudos de implantação de acessibilidade em ambiente de ensino universitário, obtidos através de pesquisa bibliográfica. Usando como recorte espacial o anel viário do Campus da Universidade Federal de Juiz de Fora, a metodologia utilizada para coleta de informações para criação de um banco de dados incluiu mapeamento por geolocalização (GPS) e endereçamento do local, cadastramento das condições ambientais existentes, classificação das rotas externas de passagem entre as edificações e criação de uma simbologia representativa dos elementos urbanos existentes voltados para acessibilidade, mobilidade e inclusão, fornecendo condições para que os usuários do Campus Universitário, sejam eles pessoas com deficiência física ou mobilidade reduzida, estrangeiros ou visitantes, tenham iguais condições de localizar os prédios, os acessos, os equipamentos urbanos e as melhores rotas para seu destino.
\end{abstract}

Palavras-chave: Mapeamento por geolocalização, endereçamento, acessibilidade. 


\begin{abstract}
There are times, technological development has influenced the ways of seeing, thinking and experiencing architecture. Every innovation, regardless of its technical area, connected to each other brings a restructuring of construction techniques and consequently the landscape. Every technological improvement has the purpose of improving the condition of human life, directly intervening in the effectiveness and efficiency of the execution of their daily activities. Humanity has witnessed the emergence of new techniques of graphic representation in the virtual environment and the development of increasingly powerful and dynamic software. The hardware had to follow all of this development and the emergence of wireless models allowed the office could be anywhere, and part of this hardware was transformed into mobile devices like phones and tablets, already regarded as important tools for construction labor. Given this context, this research aims to accomplish the use of mobile devices to facilitate accessibility within the standards of universal access, taking as a tool to create a prototype of a mobile application using geolocation technology, based on studies of implantation at room accessibility university education, obtained through literature search. Using spatial area as the main route of the Federal University of Juiz de Fora, the methodology used to collect information to create a database included mapping geolocation (GPS) and addressing the local registration of existing environmental conditions, classification external routes of passage between the buildings and the creation of a representative symbols of existing urban elements facing accessibility, mobility and inclusion, providing conditions for users of the Campus, they are people with disabilities or reduced mobility, or foreign visitors, have equal conditions to locate the buildings, accesses, urban equipment and the best routes to their destination.
\end{abstract}

Keywords: Geolocation mapping, addressing, accessibility.

\title{
1. INTRODUÇÃO
}

Fatores como a localização no eixo da Estrada Real entre Rio de Janeiro, São Paulo e Belo Horizonte, a implantação de grandes indústrias têxteis que a fizeram conhecida como a "Manchester Mineira", além de ser palco da implantação da primeira rodovia pavimentada do Brasil, impulsionaram a cidade de Juiz de Fora numa caminhada de desenvolvimento social, econômico e geográfico reposicionando-a continuamente ante as principais cidades do país.

A Universidade Federal de Juiz de Fora nasce em um contexto de grandes mudanças no país, como a inauguração da cidade de Brasília como nova sede do Distrito Federal e a reformulação do pensamento do ensino universitário no Brasil. Em seus primeiros anos foi reconhecida como "símbolo da modernidade em Juiz de Fora, tendo o seu espaço utilizado das mais diversas maneiras pela cidade destacando sempre seu caráter moderno e funcionalista" (ALBERTO, 2014).

A maneira como o projeto do campus foi concebido, adotava uma concepção dada às cidades universitárias da época e se baseava num urbanismo modernista que se propunha a humanizar as cidades estabelecendo um equilíbrio entre o individual e o coletivo, considerando que, como a maioria dos usuários do campus universitário seria de estudantes, ele deveria ser planejado em função da vida estudantil. Assim sendo, foram considerados dois setores distintos, um de vivência e outro de ensino e pesquisa (ALBERTO, 2014). 
Passados 43 anos da inauguração do campus da UFJF, é notório o desenvolvimento da universidade no aumento do número de cursos de graduação e pós-graduação, consequentemente de alunos, professores, plataformas, vias de acesso e edificações. Toda essa nova demanda e as novas formas de se ver e projetar arquitetura distanciaram a estrutura arquitetônica e urbanística do campus atual de sua concepção original, tornando-o monumental em seu conjunto, mas tendo cada elemento arquitetônico pensado individualmente, baseado na estruturação política que se tornou um forte obstáculo à implementação de um plano diretor e gerou uma grande barreira de acessibilidade considerando o grau de dificuldade de localização dos seus ambientes.

\section{A ACESSIBILIDADE EM AMBIENTES DE ENSINO UNIVERSITÁRIO}

Ao se fazer uma reflexão sobre dificuldades de acesso, o primeiro elemento a ser ponderado é a barreira física arquitetônica, que surge como obstáculos construídos nas edificações ou no próprio meio urbano, impedindo ou dificultando a livre circulação das pessoas que vivem com alguma limitação, seja ela transitória ou permanente (BITTENCOURT et al., 2004).

Esta reflexão deve considerar que muitos dos elementos arquitetônicos existentes em um campus universitário foram construídos em épocas não muito recentes, "quando $o$ paradigma da inclusão na filosofia do desenho universal não era concebido pela arquitetura dos edificios públicos" (BORGA et al., 2013), e nem ao menos se considerava a presença de alunos, professores ou servidores com deficiência.

Outro ponto a ser considerado nesta reflexão sobre acessibilidade em ambientes de ensino universitário é que há certos elementos que se tornam barreiras de acessibilidade para qualquer pessoa, ainda que não possua nenhuma deficiência ou problemas por mobilidade reduzida. A sinalização inadequada ou inexistente é um fator importante e que impede a muitos de chegarem ao seu destino, independente de sua condição física, de maneira que se tornam importantes as ações e intervenções que visem à adequações e adaptações desses espaços (BORGA et al., 2013). Esta pesquisa visa analisar o quanto um aplicativo móvel é capaz de interferir na eficiência e na eficácia de um projeto sinalético com fins de acessibilidade. O campus da UFJF, assim como tantos outros, tem entre seus usuários: idosos, pessoas com deficiência e/ou mobilidade reduzida, estrangeiros e visitantes. Um ambiente construído, quando acessível a todos, é capaz de oferecer oportunidades igualitárias a todos os seus usuários (BITTENCOURT et al., 2004).

Para assessorar esta pesquisa, foi feita uma análise dos processos de mapeamento da acessibilidade nos campi de importantes universidades brasileiras e estrangeiras, cuja seleção se deu entre aquelas que possuíam um plano de acessibilidade ou uma equipe ou departamento de estudos relativos à acessibilidade em seu campus, conforme descrito abaixo:

.1 Universidade Federal de Minas Gerais - UFMG: Possui o laboratório ADAPTESE, da Escola de Arquitetura; através de um levantamento das condições ambientais, fez uma categorização de diferentes rotas externas de passagens entre edificações; criou uma simbologia representativa de diferentes 
situações; representou as informações coletadas em mapas impressos e interativos e desenvolveu projeto de mapas de acessibilidade no campus, disponibilizando-os em diversas mídias como áudio e gráficos tridimensionais e fotografias para atender a um público variado independente da condição física ou habilidades incomuns (PICCELLI et al, 2008).

.2 Universidade Federal do Rio de Janeiro - UFRJ: Possui o núcleo Pró-Acesso; desenvolveu uma pesquisa intitulada "Acessibilidade aos Espaços de Ensino Público", com ênfase em Desenho Universal no campus da UFRJ; sua metodologia de coleta de dados concentrou-se, em grande parte, na identificação de certas atividades da vida cotidiana exercidas por pessoas com deficiência. Esta pesquisa partiu da hipótese de que as pessoas com deficiência são segregadas devido aos espaços inacessíveis, tendo, portanto o seu desempenho prejudicado no aprendizado e nas atividades de pesquisa; os autores desta pesquisa encontraram muita dificuldade em coletar dados a partir do depoimento dos usuários com deficiência, em alguns casos por descrédito de que a Universidade faria algum tipo de intervenção a seu favor e em outros casos por acharem que seriam tratados como meros objetos de estudo. A coleta de dados a partir do levantamento de campo implicou na visita autorizada a todas as dependências dos edifícios, considerando os que possuem em seu acesso escadas, degraus ou rampas muito inclinadas, a existência ou não de vagas especiais no estacionamento e sua devida sinalização, a existência de carpetes, tapetes ou irregularidades no piso impossibilitando o acesso, largura das portas e dos corredores, assim como dos espaços de ensino como salas de aula, laboratórios, bibliotecas, centrais de informática, etc; A pesquisa do Núcleo Pró-acesso no campus da UFRJ concluiu que a inadequação dos espaços e acessos impediam que pessoas com deficiência pudessem frequentar a universidade ou levar uma vida acadêmica com autonomia (DUARTE e COHEN, 2004).

.3 Universidade de São Paulo - USP - Campus Bauru: Possui uma Comissão para assuntos relativos à pessoas com deficiência, com atribuição de diagnosticar as barreiras arquitetônicas, propor intervenções para adequação dos espaços internos e externos do campus da USP e promover ações de conscientização da comunidade acadêmica. Possui o Programa USP Legal com a missão de elaborar políticas internas de inserção de pessoas com deficiência no ambiente universitário. A escolha deste campus se deu pelo fato de ser um estabelecimento público, prioritariamente da área de saúde e totalmente voltado para o ensino, pesquisa e assistência à comunidade. A Comissão fez um mapeamento de barreiras físicas e intervenções executadas, obtendo dados por meio de percurso das áreas internas e acessos externos, realizando uma pesquisa somente descritiva e quantitativa cujos resultados ainda não foram utilizados na sua totalidade para implementação de ações de acessibilidade. (LAMONICA et al, 2008)

.4 Universidade Estadual de Campinas - UNICAMP: Possuidora do maior núcleo de acessibilidade do país, cujo objetivo visa acesso, permanência e prosseguimento da escolaridade de nível superior de pessoas com deficiência através da criação de ambientes inclusivos. Possui o Laboratório de Acessibilidade - LAB - para onde convergem os trabalhos de diversos grupos de pesquisadores da UNICAMP, compondo assim o grupo Todos Nós - UNICAMP acessível através do qual diversas ações são efetuadas com o intuito de atrair, inserir e manter com dignidade e 
autonomia o estudante e o servidor com deficiência. (PUPO et al, 2004). Este projeto conta também com uma vasta produção acadêmica, cujas linhas de atuação perpassam pelos seguintes tópicos: Atendimento Educacional Especializado, Ensino Inclusivo, Acesso ao Conhecimento e à Tecnologia, Tecnologia Assistiva, Interação Humano-Computador e Ambientes Inclusivos de Aprendizagem (MANTOAN e BARANAUSKAS, 2006). Dentre as ações voltadas para facilitar o acesso ao ambiente construído, pode-se citar a criação de maquetes táteis e sonoras (Figura 10), tendo por finalidade orientar e descrever o ambiente para pessoas com deficiência visual, dois projetos infra-estruturais compostos por uma sala de acesso à informação e o laboratório de apoio didático (LAB), ambientes projetados para serem utilizados não apenas por pessoas com deficiência, extrapolando o design especializado. Pode-se citar também a criação de um aplicativo móvel chamado "Unicamp Serviços" (Figura 11), que oferece as seguintes funcionalidades:

- Consulta a pontos de interesse (geolocalização);

- Consulta à lista de ramais da Unicamp;

- Saldo do cartão dos alunos (R.U. e Biblioteca);

- Cardápio dos Restaurantes;

- Horários e trajetos do ônibus circular interno e da moradia estudantil;

- Consulta, reserva e renovação do acervo da biblioteca;

- Consulta aos agendamentos feitos na Odontologia e na Clínica Médica para alunos, docentes e funcionários;

- Serviço de informações ao cidadão Unicamp, para solicitar documentos e dados relativos à Unicamp, de acordo com a Lei de Acesso à Informação, instituída pela Lei Federal no 12.527, de 18 de novembro de 2011;

- Notícias do Campus (Jornal da Unicamp, Agenda de Eventos e Teses do Mês);

- Solicitações administrativas;

- Acesso ao Portal do Centro de Computação da Unicamp e ao Portal Unicamp.

Dentre as estrangeiras, foram analisados os programas e ações de acessibilidade das Universidades Ohio University, North Carolina State University e University of Missouri, que tem como particularidade a utilização de mapas de acessibilidade, que retratam o bom endereçamento dos campi e demonstram de forma clara e com simbologia específica os espaços acessíveis, inacessíveis e vazios, as rotas acessíveis, difíceis e inacessíveis, os estacionamentos e as vagas especiais, além da localização dos equipamentos e mobiliários urbanos e a marcação das rotas para se fazer à pé.

As Universidades analisadas como referência possuem diferentes formas de pensar a problemática da acessibilidade em ambiente de ensino universitário e serviram de base para que esta pesquise fizesse uma reflexão sobre o quanto o uso de um aplicativo móvel pode complementar e aumentar a eficácia e a eficiência de um bom endereçamento e uma boa sinalização. 


\section{METODOLOGIA DE COLETA DE DADOS}

\subsection{Mapeamento}

Tendo como base a Planta Geral do campus da UFJF, partiu-se da hipótese de que a dificuldade de localização dos ambientes é uma forte barreira de acessibilidade e que um mapeamento das condições existentes, aliado à criação de um endereçamento e à uniformização da comunicação visual quebraria esta dificuldade. A segunda hipótese é que a soma da tecnologia de um aplicativo móvel à essas mudanças traz mais eficiência ao processo. O Anel Viário, utilizado como recorte espacial desta pesquisa, teve todo o seu trajeto percorrido para conferência e atualização das plantas e elementos cadastrados. O Anel Viário possui os dois principais acessos ao campus universitário: o Pórtico Norte com acesso pelo Bairro São Pedro e o Pórtico Sul com acesso pelo Bairro Dom Bosco. Ele é o ponto de partida para todos os setores da UFJF, além de ser o local com o maior trânsito de visitantes, uma vez que os ônibus municipais trafegam dentro do campus e é o local com o maior fluxo de veículos, pois serve de passagem entre bairros. Ele é o lugar onde acontecem os eventos de maior impacto ambiental e envolvimento social da Universidade.

Este mapeamento detectou que o Anel Viário possui os seguintes dados:

- 07 entradas de estacionamento sendo uma na primeira plataforma, com 92 vagas, uma no Centro Olímpico, com 109 vagas, uma no Corpo de Bombeiros com 05 vagas, uma no lado direito da plataforma de Estudos Sociais com 113 vagas, uma no lado esquerdo da plataforma de Estudos Sociais com 112 vagas, sendo duas reservadas para pessoa com deficiência, uma na Reitoria, com 246 vagas, sendo quatro reservadas para pessoa com deficiência e uma na PróReitoria de Infraestrutura, com 51 vagas, sendo uma reservada para pessoa com deficiência;

- 06 pontos de ônibus (internos e municipais);

- 09 travessias de pedestres através de Traffic Calming;

- 01 bicicletário (Reitoria);

- 04 orelhões em perfeito estado de conservação;

- 02 sanitários químicos;

- 09 elevadores;

- 08 secretarias e;

- 05 Infocentros.

Entre os equipamentos urbanos, possui:

- Farmácia Universitária;

- Escola Municipal Santana Itatiaia;

- Corpo de Bombeiros;

- Centro de Vivência com Observatório Astronômico, Concha Acústica, Skatepark, ginástica ao ar livre e um restaurante;

- 02 cantinas; 
- 02 Agências Bancárias e 03 Caixas Eletrônicos independentes;

- Biblioteca Central;

- Sede da Associação de Professores de Ensino Superior;

- Sede do Diretório Central de Estudantes;

- Central Telefônica;

- Secretaria Avançada do Sindicato dos Trabalhadores em Educação da UFJF;

- Centro de Pesquisas Sociais;

- Reitoria;

- 03 anfiteatros e;

- 01 Lago.

No Anel Viário também estão instaladas 05 Faculdades e 01 Instituto e dá acesso para outras 08 Plataformas e suas instalações.

\subsection{Cadastramento das condições ambientais existentes}

O cadastramento das condições ambientais existentes levou em consideração a definição de rota acessível como "um conjunto de medidas de acessibilidade que visam tornar um determinado espaço completamente acessível.” (DUARTE et al, 2004)

Este cadastramento foi feito através de relatório escrito e fotográfico e visa fornecer dados que auxiliarão ao aplicativo e seu usuário na escolha da melhor rota a ser seguida, considerando a existência de qualquer tipo de barreira, por menor que pareça, uma vez que "um único elemento que se caracterize como uma barreira física pode invalidar todas as outras medidas de acessibilidade adotadas. " (DUARTE et al, 2004)

A forma de cadastramento deverá ser interativa e contínua. Quando o usuário se deparar com obstáculos na sua rota de acesso, deverá fotografar e enviar aos administradores do aplicativo, para que seja feita alteração dos mapas e traçado das rotas. Desta forma, as informações se manterão atualizadas e ações de intervenção poderão ser realizadas para solucionar o problema.

\subsection{Geolocalização}

Denomina-se geoprocessamento a ação de utilizar-se de técnicas matemáticas e computacionais para o tratamento da informação geográfica, e que influencia diretamente em diversas áreas ligadas à engenharia civil e ambiental, como: cartografia, análise de recursos naturais, agricultura de precisão, transportes, comunicação, energia e planejamento urbano. Trata-se de um conjunto de tecnologias de coleta, tratamento, manipulação e apresentação de informações espaciais, voltado para um objetivo específico (BERNARDI \& LANDIM, 2002).

Esta pesquisa apossou-se da aplicação do sistema de posicionamento global (GPS) para a coleta de dados fundamentais para a criação de um novo endereçamento no campus universitário.

O mapeamento por geolocalização foi feito com dispositivos móveis e com o auxílio do software GPS TrackMaker. Nele foram compilados os dados obtidos no levantamento 
de campo e, com o apoio das plantas em AutoCAD foram feitas as marcações dos pontos de interesse a serem lidos pelo aplicativo em desenvolvimento.

\section{O ENDEREÇAMENTO ALIADO À INFORMAÇÃO}

\subsection{Conceituando endereçamento}

Considerando que a sinalização inadequada ou inexistente gera dificuldade de localização de um ambiente e consequentemente uma barreira de acessibilidade, para eficácia desta pesquisa, fez-se necessária a criação de um endereçamento no local objeto deste estudo, denominando a rua que forma o Anel Viário e as ruas diretamente ligadas a ele, denominar e numerar cada prédio existente e todas as salas que o compõem.

Através de um endereçamento é possível melhorar a localização das edificações e ambientes e a orientação de seus usuários; facilitar as intervenções de emergência seja por ambulâncias, bombeiros ou polícia; situar os serviços prestados e facilitar a gestão ambiental (GODIN, 2005).

O endereçamento proposto não consta de uma simples operação sinalética, mas um mapeamento detalhado possibilitando o recolhimento de informações morfológicas, históricas e conceituais, facilitando não somente a localização da edificação, mas também sua manutenção, reforma ou alteração do uso. A forma de endereçamento proposta visa ser um importante instrumento de programação e gestão dos serviços técnicos por facilitar o conhecimento do patrimônio público através do seu histórico de saneamento, instalações prediais, estrutura, acabamento, tecnologia aplicada, histórico das intervenções, redes, etc.

Para que isto ocorra, além de letreiros e números nas fachadas e nas portas, esta pesquisa propõe a implantação de um código de barras $2 \mathrm{D}$ ou $\mathrm{QR}$ Code em todas as fachadas, com a finalidade de indicar onde a pessoa se encontra e prestar informações mais detalhadas do local através do uso de dispositivos móveis.

Recém surgida e amplamente disseminada no mundo corporativo, o código de barras bidimensional, também chamado 2D ou QR-Code é lido por câmeras de dispositivos móveis como celulares e tablets com o auxílio de um aplicativo decodificador. O QR Code é uma forma rápida, fácil e barata de obter informação, pois tem capacidade de armazenamento de 7089 caracteres numéricos, 4296 caracteres alfanuméricos, 2953 bytes e 1817 caracteres de símbolos japoneses Kanji/Kana, o que faz dele uma ferramenta de comunicação com seus usuários (JÚNIOR et al, 2012). A imagem, após captada e decodificada pelo aplicativo é transmitida como mensagem, que pode ser um dispositivo para URL, um endereço, um vídeo entre outros conteúdos (JÚNIOR et al, 2012).

Através do aplicativo móvel, o usuário tem acesso à sua localização por geoprocessamento, à localização de setores e ambientes edificados ou abertos, informações sobre como chegar através do meio de transporte que está utilizando (ônibus municipal, ônibus fretado, ônibus circular da UFJF, carro, motocicleta, bicicleta ou à pé), informações da melhor rota com base nas suas habilidades ou ausência delas, além das distâncias dos equipamentos e mobiliários urbanos e condições de uso dos mesmos. 


\section{CONCLUSÕES OU CONSIDERAÇÕES FINAIS}

Esta pesquisa pretende contribuir para a eficiência e a eficácia de ações de acessibilidade universal como endereçamento e comunicação visual, que podem ser aplicáveis em ambientes de ensino universitário e outros de semelhante dimensão, conectando estas ações através de um aplicativo móvel que atuará como um facilitador na implementação deste processo ao distinguir ambientes, rotas, facilitando a leitura do ambiente construído e propondo alternativas que interferem nas barreiras técnicas e paulatinamente nas barreiras atitudinais.

De igual forma, esta pesquisa visa contribuir com os processos de construção de um plano de acessibilidade ao campus universitário da UFJF ao propor formas de endereçamento e identificação dos ambientes, fornecendo informações precisas através do mapeamento de seus acessos e elementos urbanísticos de apoio.

Por outro lado, pretende-se levantar discussões referentes ao (des)preparo dos atuais usuários das universidades ao receber uma nova demanda de usuários com necessidades especiais, sabendo que uma universidade, além de profissionais altamente qualificados, precisa formar cidadãos.

\section{REFERÊNCIAS}

ALBERTO, K. C. Os debates para a criação e consolidação da Universidade Federal de Juiz de Fora (1930-1960). 2014. Anais do XI Congreso Iberoamericano de Historia de la Educación Latinoamericana. Toluca, México.

ASSOCIAÇÃO BRASILEIRA DE NORMAS TÉCNICAS (ABNT). NBR-9050: Acessibilidade a edificações, mobiliário, espaços e equipamentos urbanos. Rio de Janeiro: ABNT, 2004.

BERNARDI, J.V.E. \& LANDIM, P.M.B. Aplicação do Sistema de Posicionamento Global (GPS) na coleta de dados. 2002. DGA, IGCE, UNESP/Rio Claro, Lab. Geomatemática, Texto Didático 10, 31 pp. 2002. Disponível em $<$ http://www.rc.unesp.br/igce/aplicada/textodi.html>. Acesso em: 14/03/2014

BITTENCOURT, L.S., CORRÊA, A.L.M., MELO, J.D., MORAES, M.C., RODRIGUES, R.F. Acessibilidade e Cidadania: Barreiras Arquitetônicas e Exclusão Social dos Portadores de Deficiências Físicas. 2004. Anais do $2^{\circ}$ Congresso Brasileiro de Extensão Universitária. Belo Horizonte, Minas Gerais.

BORGA, K., OLIVEIRA, G.F., BRIDI, L.R.T. 2013. Acessibilidade em ambiente de ensino: identificação de barreiras arquitetônicas no IFC Campus Friburgo. Mostra Nacional de Iniciação Científica e Tecnológica Interdisciplinar - VI MIC TI. Instituto Federal Catarinense - Campus Camboriú - SC.

DUARTE, Cristiane Rose de Siqueira ; COHEN, R. . Acessibilidade aos Espaços do Ensino e Pesquisa: Desenho Universal na UFRJ - Possível ou Utópico? In: NUTAU 2004: Demandas Sociais, Inovações Tecnológicas e a Cidade, 2004, Sâo Paulo. Anais NUTAU 2004: Demandas Sociais, Inovações Tecnológicas e a Cidade, 2004.

CREA-MG. Guia de acessibilidade urbana. Belo Horizonte - MG. 2006

GODIN, L., FARVACQUE-VITKOVIC, C., LEROUX, H., VERDET, F., CHÁVEZ, R. 2005. O endereçamento e a gestão das cidades. Banco Internacional para 
Reconstrução e Desenvolvimento / Banco Mundial 1818 H Street, N.W., Washington, D.C.

JÚNIOR, D.L., LOTERIO, G.A., MAGNANI, L.E., SILVA, F.N.M. O uso de códigos de barras bidimensionais (QR-Codes) como ferramentas para ampliação da experiência publicitária: um estudo sobre os anúncios veiculados em um jornal brasileiro. 2012. Anais do XV SEMEAD - Seminários em Administração. FEA - USP. São Paulo, SP.

LAMÔNICA , Dionísia Aparecida Cusin; ARAÚJO-FILHO, Pedro ; SIMOMELLI, Simone Berriel Joaquim; CAETANO, Vera Lígia Santos Butiquiol; REGINA, Márcia Regina Rodrigues; REGIANI, Denise Maria. 2008. Acessibilidade em ambiente universitário: identificação de barreiras arquitetônicas no campus da USP de Bauru. Rev. Bras. Ed. Esp., Marília, Mai.-Ago. 2008, v.14, n.2, p.177-188

MANTOAN, M.T.E., BARANAUSKAS, M.C. Todos Nós - Unicamp Acessível. In: Diferentes Contextos de Educação Especial/Inclusão Social / PROESP, 2006, Santa Maria:Pallotti. p. 45-60.

NETO, R.S.N., RUSCHEL, R.C., PICCHI, F.A. Avaliação de ferramentas de Tecnologia da Informação na construção com funcionalidades móveis compatíveis aos itens da NBR ISSO 9001:2008. 2012. REEC - Revista Eletrônica de Engenharia Civil. Volume6, $\mathrm{n}^{\mathrm{o}}$ 1. Disponível em: http://revistas.ufg.br/index.php/reec/index Acessado em 14/02/2014.

PICCELI, Angélica Fátima Baldin; GRENFELL, Camilla Fortini Pinto; GUIMARÃES, Marcelo Pinto. O Processo de Mapeamento da Acessibilidade no Campus da UFMG. Laboratório ADAPTSE, Escola de Arquitetura. UFMG. Belo Horizonte - MG. 2008 .

PUPO, D.T., BONILHA, F.F.G., CARVALHO, S.H.R. Laboratório de Acessibilidade: Criação, Implantação e Inclusão de Pessoas com Necessidades Educacionais Especiais na Biblioteca Central da UNICAMP. Anais do XIII Seminário Nacional de Bibliotecas Universitárias - SINBU. Natal - RN. 2004.

TONiazzO, J. C., PAZETO, T. A., JACOSKI, C. A. 2006. Protótipo para Coleta e Transmissão de Dados na Construção Civil Utilizando Dispositivos Móveis. 2006. XII Encontro Nacional de Tecnologia no Ambiente Construído - ENTAC. Florianópolis, SC. 